\title{
An Extension of the Spectral Mapping Theorem
}

\author{
A. R. Medghalchi ${ }^{1}$ and S. M. Tabatabaie ${ }^{2}$ \\ ${ }^{1}$ Faculty of Mathematical Sciences and Computer Engineering, Tabbiat Moallem University, \\ Tehran 15618, Iran \\ ${ }^{2}$ Department of Mathematics, The University of Qom, Qom 3716146611, Iran
}

Correspondence should be addressed to S. M. Tabatabaie, aylar5@yahoo.com

Received 8 January 2008; Accepted 4 May 2008

Recommended by Ingo Witt

We give an extension of the spectral mapping theorem on hypergroups and prove that if $K$ is a commutative strong hypergroup with $\widehat{K}=X_{b}(K)$ and $\kappa$ is a weakly continuous representation of $M(K)$ on a $W^{*}$-algebra such that for every $t \in K, \kappa_{t}$ is an $*$-automorphism, sp $\kappa$ is a synthesis set for $L^{1}(K)$ and $\kappa\left(L^{1}(K)\right)$ is without order, then for any $\mu$ in a closed regular subalgebra of $M(K)$ containing $L^{1}(K), \sigma(\kappa(\mu))=\overline{\widehat{\mu}(s p \kappa)}$, where sp $\kappa$ is the Arveson spectrum of $\kappa$.

Copyright ( 2008 A. R. Medghalchi and S. M. Tabatabaie. This is an open access article distributed under the Creative Commons Attribution License, which permits unrestricted use, distribution, and reproduction in any medium, provided the original work is properly cited.

\section{Introduction and notation}

Hypergroups were introduced in a series of papers by Jewett [1], Dunkle [2], and Spector [3] in 70's. They are in fact extensions of topological groups with the difference that hypergroups do not have necessarily an algebraic structure. Roughly speaking, the product of two elements of a hypergroup is a probability measure. A hypergroup is a locally compact space which has enough structure so that a convolution on the space of finite regular Borel measures can be defined. Therefore, the extension of Fourier analysis on hypergroups is made with more difficulties and usually with different proofs in the group case. Classical examples of hypergroups are locally compact groups, the space of conjugacy classes of a compact group, spaces of orbits in the group of automorphisms, and double-cosets of certain nonnormal closed subgroups of a compact group. We will state definition and some basic properties of hypergroups in Section 2. Throughout this paper, $K$ is a commutative strong hypergroup with $\widehat{K}=X_{b}(K)$. In Section 4, we give some examples of this type of hypergroups. We denote by $M(K)$ the space of all regular complex Borel measures on $K$, by $M^{+}(K)$ the subset of positive measures in $M(K)$, and by $\delta_{x}$ the Dirac measure at the point $x$.

A $C^{*}$-algebra $M$ is called $W^{*}$-algebra if for a Banach algebra $M_{*},\left(M_{*}\right)^{*}=M$. Any $W^{*}-$ algebra is unitary (with unit $1_{M}$ ). The famous examples of $W^{*}$-algebras are von Neumann 
algebras. We can consider $\sigma\left(M, M_{*}\right)$ (i.e., $w k^{*}$ ) topology on $M$ [4]. In this paper, $M$ is always a $W^{*}$-algebra. We denote by $B_{\sigma}(M)$ the set of all $\left(\sigma\left(M, M_{*}\right), \sigma\left(M, M_{*}\right)\right)$-continuous operators on $M$.

Let $\kappa: M(K) \rightarrow B_{\sigma}(M)$ be a norm-decreasing algebra-homomorphism. For any $t \in K$, we denote $\kappa_{t}=\kappa\left(\delta_{t}\right)$. Suppose that $\kappa$ has the following properties.

(1) For any $t \in K, \kappa_{t}: M \rightarrow M$ is an $*$-automorphism. (Then by [5, Theorem 4.8, page 253] any $\mathcal{K}_{t}$ is an isometry.)

(2) For any $x \in M$ and $\rho \in M_{*}$, the function $t \mapsto\left\langle\kappa_{t}(x), \rho\right\rangle$ is continuous.

(3) $\kappa_{e}=I_{M}$, where $e$ is the identity of $K$ and $I_{M}$ is the identity mapping on $M$.

Let $x \in M$ and $\rho \in M_{*}$. Obviously for any measure $v \in M(K)$ with finite support, we have $\langle\kappa(v)(x), \rho\rangle=\int_{K}\left\langle\kappa_{t}(x), \rho\right\rangle d v(t)$. Let $\mu \in M(K)$. Since the set $E$ containing all measures in $M(K)$ that have finite support is dense in $M(K)$, there exists a net $\left(v_{\beta}\right) \subseteq E$ such that $v_{\beta} \rightarrow \mu$ in $M(K)$. Then by [1, Lemma 2.2C], we have $\int_{K} f d v_{\beta} \rightarrow \int_{K} f d \mu$, where $f(t)=\left\langle\kappa_{t}(x), \rho\right\rangle(t \in K)$. On the other hand, by continuity of $\mathcal{\kappa}, \int_{K} f d v_{\beta}=\left\langle\kappa\left(v_{\beta}\right)(x), \rho\right\rangle \rightarrow\langle\kappa(\mu)(x), \rho\rangle$. Then, for any $\mu \in M(K)$ we have

$$
\langle\kappa(\mu)(x), \rho\rangle=\int_{K}\left\langle\kappa_{t}(x), \rho\right\rangle d \mu(t),
$$

where $x \in M$ and $\rho \in M_{*}$.

\section{Basic properties of hypergroups}

First, we recall the definition and basic properties of a hypergroup. The main references are $[1,6]$.

Definition 2.1. Let $K$ be a locally compact Hausdorff space. The space $K$ is a hypergroup if there exists a binary mapping $(x, y) \mapsto \delta_{x} * \delta_{y}$ from $K \times K$ into $M^{+}(K)$ satisfying the following conditions.

(1) The mapping $\left(\delta_{x}, \delta_{y}\right) \mapsto \delta_{x} * \delta_{y}$ extends to a bilinear associative operator $*$ from $M(K) \times M(K)$ into $M(K)$ such that

$$
\int_{K} f d(\mu * v)=\int_{K} \int_{K} \int_{K} f d\left(\delta_{x} * \delta_{y}\right) d \mu(x) d v(y)
$$

for all continuous functions $f$ on $K$ vanishing at infinity.

(2) For each $x, y \in K$ the measure $\delta_{x} * \delta_{y}$ is a probability measure with compact support.

(3) The mapping $(\mu, v) \mapsto \mu * v$ is continuous from $M^{+}(K) \times M^{+}(K)$ into $M^{+}(K)$; the topology on $M^{+}(K)$ being the cone topology.

(4) There exists an $e \in K$ such that $\delta_{e} * \delta_{x}=\delta_{x}=\delta_{x} * \delta_{e}$, for all $x \in K$.

(5) There exists a homeomorphism involution $x \mapsto x^{-}$from $K$ onto $K$ such that, for all $x, y \in K$, we have $\left(\delta_{x} * \delta_{y}\right)^{-}=\delta_{y^{-}} * \delta_{x^{-}}$, where for $\mu \in M(K), \mu^{-}$is defined by

$$
\int_{K} f(t) d \mu^{-}(t)=\int_{K} f\left(t^{-}\right) d \mu(t),
$$


and also,

$$
e \in \operatorname{supp}\left(\delta_{x} * \delta_{y}\right) \quad \text { iff } y=x^{-},
$$

where $\operatorname{supp}\left(\delta_{x} * \delta_{y}\right)$ is the support of the measure $\delta_{x} * \delta_{y}$.

(6) The mapping $(x, y) \mapsto \operatorname{supp}\left(\delta_{x} * \delta_{y}\right)$ from $K \times K$ into the space $\mathbf{C}(K)$ of compact subsets of $K$ is continuous, where $\mathrm{C}(K)$ is given the topology whose subbasis is given by all

$$
\mathrm{C}_{U, V}=\{A \in \mathbf{C}(K): A \cap U \neq \varnothing, A \subseteq V\}
$$

where $U, V$ are open subsets of $K$.

Note that $\delta_{x} * \delta_{y}$ is not necessarily a Dirac measure. A hypergroup $K$ is commutative if $\delta_{x} * \delta_{y}=\delta_{y} * \delta_{x}$, for all $x, y$ in $K$. Each commutative hypergroup $K$ carries a Haar measure $m$ such that $\delta_{x} * m=m$, for all $x \in K$, as shown by Spector [7]. In any commutative hypergroup $K$, we have $m=m^{-}$(see [1, Section 5.3]). Let $f, g$ be Borel functions on $K$ and $\mu \in M(K)$. For any $x, y \in K$ we denote $f_{x}(y)=f(x * y):=\int_{K} f d\left(\delta_{x} * \delta_{y}\right)$. Also we define

$$
(\mu * f)(x):=\int_{K} f\left(y^{-} * x\right) d \mu(y), \quad(f * g)(x):=\int_{k} f(x * y) g\left(y^{-}\right) d m(y),
$$

where $x \in K$. If $x, y \in K$ and $A, B \subseteq K$, we denote $\{x\} *\{y\}=\operatorname{supp}\left(\delta_{x} * \delta_{y}\right)$, and $A * B=$ $\bigcup_{x \in A, y \in B}\{x\} *\{y\}$.

A complex-valued continuous function $\xi$ on $K$ is said to be multiplicative if $\xi(x * y)=$ $\xi(x) \xi(y)$ holds for all $x, y \in K$. The space of all multiplicative functions on $K$ is denoted by $X_{b}(K)$. A nonzero multiplicative function $\xi$ on $K$ is called a character if $\xi\left(x^{-}\right)=\overline{\xi(x)}$, for all $x$ in $K$. The dual $\widehat{K}$ of $K$ is the locally compact Hausdorff space of all characters with the topology of uniform convergence on compacta. In general, $\widehat{K}$ is not necessarily a hypergroup. A hypergroup $K$ is called strong if its dual $\widehat{K}$ is also a hypergroup with complex conjugation as involution, pointwise product as convolution, that is

$$
\eta(x) \chi(x)=\int_{\widehat{K}} \xi(x) d \delta_{\eta} * \delta_{X}(\xi),
$$

for all $\eta, x \in \widehat{K}$ and $x \in K$, and has the constant function 1 as the identity element.

For any $p>0$, we denote $L^{p}(K)=L^{p}(K, m)$ and $L^{p}(\widehat{K})=L^{p}(\widehat{K}, \pi)$, where $\pi$ is the Plancherel measure on $\widehat{K}$ associated with $m$. The structure space $\Delta\left(L^{1}(K)\right)$ of Banach algebra $L^{1}(K)$ doses not necessarily equal with $\widehat{K}$ and we only have $\Delta\left(L^{1}(K)\right)=X_{b}(K)$, while $\widehat{K} \subseteq$ $X_{b}(K)$.

For any $f \in L^{1}(K)$ and $\mu \in M(K)$, the Fourier-Stieltjes transform $\widehat{\mu}$ of $\mu$ and the Fourier transform $\hat{f}$ of $f$ are defined by

$$
\widehat{\mu}(\xi)=\int_{K} \overline{\xi(t)} d \mu(t), \quad \widehat{f}(\xi)=\int_{K} \overline{\xi(t)} f(t) d m(t),
$$

where $\xi \in \widehat{K}$. For any $f, g \in L^{2}(K)$, we have $\widehat{f}, \widehat{g} \in L^{2}(\widehat{K})$ and $\widehat{f} * \widehat{g}=\widehat{f g}[6]$. 


\section{The main result}

Recall that for any complex commutative algebra $A, \Delta(A)$, the structure space of $A$, is a locally compact Hausdorff space with Gelfand topology and for each $a \in A$, the Gelfand transform $\widehat{a}$ is in $C_{0}(\Delta(A))$. For an ideal $I$ of $A$, the hull of $I$ is defined by $h(I)=\xi \in \Delta(A): \widehat{a}(\xi)=0$ for every $a \in I\}$. For each closed $F \subseteq \Delta(A)$, we denote $k(F)=\{a \in A: \widehat{a}(\xi)=0$ for every $\xi$ in $F\}$ and $J_{0}(F)=\{a \in A: \widehat{a}=0$ on some neighborhood of $F$, and $\operatorname{supp}(\widehat{a})$ is compact $\}$, where $\operatorname{supp}(\widehat{a})$ is the support of the function $\widehat{a}$. We denote $J(F)=\overline{J_{0}(F)}$, the closure of $J(F)$. For any Banach algebra $A$, the spectrum of any $a \in A$ is denoted by $\sigma_{A}(a)$.

Definition 3.1. The Arveson spectrum of $\kappa$ is defined by

$$
\begin{aligned}
s p \mathcal{\kappa} & :=h\left(\left\{f \in L^{1}(K): \kappa(f)=0\right\}\right) \\
& =\left\{\xi \in \widehat{K}: \text { for any } f \in L^{1}(K), \kappa(f)=0 \text { implies } \widehat{f}(\xi)=0\right\} .
\end{aligned}
$$

The Arveson spectrum and spectral subspaces on hypergroups have been studied in [8]. In this paper, we concentrate on an extension of the spectral mapping theorem to hypergroups. The spectral mapping theorem gives the relation $\sigma(\kappa(\mu))=\overline{\widehat{\mu}(s p \kappa)}$ for some measures $\mu \in M(K)$. In the case that $K$ is a locally compact Abelian group, Connes proved the spectral mapping theorem for every Dirac measure $\mu$ [9]. Then, D'Antoni et al. proved that the spectral mapping theorem holds for those measures whose continuous part belongs to $L^{1}(G)$ [10]. Furthermore, Eschmeier proved the spectral mapping theorem for $\mathcal{\kappa}$ as the translation group representation, in the case that $M$ is Banach algebra $L^{1}(G)$ or $M(G)$ and the convolution operator induced by $\mu$ has the weak 2-SDP [11]. Takahasi and Inoue proved the spectral mapping theorem for any regular subalgebra of $M(G)$ in the case that $G$ is compact [12]. Also, Seferoglu proved that $\sigma(\kappa(\mu))=\overline{\widehat{\mu}(\operatorname{spec}(\kappa))}$ holds when $\kappa\left(L^{1}(G)\right)$ is without order and sp $\kappa$ is a synthesis set for $L^{1}(G)$ [13]. (A subset $C$ of Banach algebra $A$ is called without order if for all $a \in A, a . C=\{0\}$ implies $a=0[14]$.

A Banach algebra $A$ is called regular if for any closed subset $E$ of $\Delta(A)$ and any $\varphi \in$ $\Delta(A) \backslash E$, there is an $a \in A$ such that $\widehat{a}(\varphi)=1$ and $\widehat{a} \equiv 0$ on $E$. For any commutative and strong hypergroup $K$ with $\widehat{K}=X_{b}(K), L^{1}(K)$ is a regular Banach algebra [15]. Also, since the Fourier transform ${ }^{\wedge}: L^{1}(K) \rightarrow C_{0}(\widehat{K})$ is a norm-decreasing $*$-homomorphism, $L^{1}(K)$ is semisimple. If $A$ is a regular commutative Banach algebra, then for any closed $F \subseteq \Delta(A), h(J(F))=F$. If $A$ is also semisimple, then $k(F)$ and $J(F)$ are the largest closed ideal and the smallest ideal with hull equal to $F$, respectively [16]. $F$ is called a set of spectral synthesis if and only if $J(F)=k(F)$. Then, for such sets, $k(F)$ is the only closed ideal in $A$ such that its hull is $F$.

It is well known that the correspondence $F \mapsto h(k(F))$ is a proper closure operation and that the topology on $\Delta(A)$ determined by this operation, called hull-kernel topology, is weaker than the Gelfand topology [17]. A commutative Banach algebra $A$ is regular if and only if the Gelfand and the hull-kernel topologies coincide on $\Delta(A)$ (see [18, Theorem 3.2.10]). Another property of commutative regular semisimple Banach algebras appears in the following lemma. It is given here for the convenience of the reader.

Lemma 3.2. Let $A$ be a commutative regular semisimple Banach algebra, let $B$ be a unitary Banach algebra (with unit $1_{B}$ ), and let $\kappa: A \rightarrow B$ be a continuous one-to-one homomorphism. 
(i) If $A$ is unitary with unit $1_{A}$ and $\kappa\left(1_{A}\right)=1_{B}$, then for any $a \in A$,

$$
\sigma_{B}(\kappa(a))=\widehat{a}(\Delta(A)) .
$$

(ii) If $A$ is without unit, then for any $a \in A$,

$$
\sigma_{B}(\kappa(a))=\overline{\widehat{a}(\Delta(A))}
$$

Proof. See [13, Lemmas 1 and 2] for the proof.

Lemma 3.3. Suppose that $\kappa: M(K) \rightarrow B(M)$ is the function introduced in Section 1. Let $\kappa\left(L^{1}(K)\right)$ be without order and let spк be a spectral synthesis set for $L^{1}(K)$. Then, $\kappa(\mu)=0$ if and only if $\left.\widehat{\mu}\right|_{s p \kappa}=0$.

Proof. Let $\mu \in M(K)$ and $\kappa(\mu)=0$. For any $f \in L^{1}(K), \mu * f \in L^{1}(K)$ and $\kappa(\mu * f)=\kappa(\mu) \kappa(f)=0$. Then

$$
\mu * f \in k h(\{\mu * f\}) \subseteq k h\left(\left\{f \in L^{1}(K): \kappa(f)=0\right\}\right)=k(s p \kappa) .
$$

So $\widehat{\mu}(\xi) \widehat{f}(\xi)=\widehat{\mu * f}(\xi)=0$, where $\xi \in s p \kappa$. For each $\xi \in \widehat{K}$, there exists a $g \in C_{c}^{+}(\widehat{K})$ such that $\xi \in \operatorname{supp}(g)$. We can consider $h \in C_{c}(K)$ such that $\widehat{h} \geq 0$ and $\widehat{h}>g$ on $\operatorname{supp}(g)$ (see [6, Proposition 2.2.5]). In other words, the set $\left\{\widehat{f}: f \in C_{c}(K)\right\}$ separates the points of $\widehat{K}$. Then, for any $\xi \in s p \kappa$ we have $\widehat{\mu}(\xi)=0$.

Conversely, let $\mu \in M(K)$ and $\left.\widehat{\mu}\right|_{s p \kappa} \equiv 0$. Then, for each $\xi \in \widehat{K}$ and $f \in L^{1}(K)$ we have $\widehat{\mu * f}(\xi)=\widehat{\mu}(\xi) \widehat{f}(\xi)=0$. Now, since $k(s p \kappa)$ is the only closed ideal in $L^{1}(K)$ whose hull is spк,

$$
\mu * f \in k(\operatorname{sp\kappa })=k h\left(\left\{f \in L^{1}(K): \kappa(f)=0\right\}\right)=\left\{f \in L^{1}(K): \kappa(f)=0\right\} .
$$

Therefore, $\kappa(\mu) \cdot \kappa\left(L^{1}(K)\right)=0$. Since $\kappa\left(L^{1}(K)\right)$ is without order, $\kappa(\mu)=0$.

Let $M_{0}(K)$ be a closed subalgebra of $M(K)$ which contains $L^{1}(K)$. Then, $\widehat{K}$ can be considered as a subset of $\Delta\left(M_{0}(K)\right)$ and for every $\mu$ in $K$, the restriction of the Gelfand transform $T \mu$ of $\mu$ to $\widehat{K}$ coincides with the Fourier-Stieltjes transform $\widehat{\mu}$ of $\mu$. This implies that $M_{0}(K)$ is a semisimple algebra.

Theorem 3.4. Suppose that $\kappa: M(K) \rightarrow B_{\sigma}(M)$ is the function introduced in Section 1. Let $\kappa\left(L^{1}(K)\right)$ be without order and let spк be a spectral synthesis set. Then, for any $\mu \in M_{0}(K)$,

$$
\sigma_{B(M)}(\kappa(\mu))=\overline{\widehat{\mu}(s p \kappa)},
$$

where $M_{0}(K)$ is a closed regular subalgebra of $M(K)$ containing $L^{1}(K)$.

Proof. Since $L^{1}(K) \subseteq M_{0}(K) \subseteq M(K), \widehat{K} \cong \Delta\left(L^{1}(K)\right)$ is a subset of $\Delta\left(M_{0}(K)\right)$. For each $\mu \in$ $M_{0}(K)$, we denote by $T \mu$ the Gelfand transform of $\mu$. We have $T \mu \in C_{0}\left(\Delta\left(M_{0}(K)\right)\right)$ and $\widehat{\mu}=$ $\left.T \mu\right|_{\widehat{K}}$. The function $\tilde{\kappa}: M_{0}(K) / I_{\mathcal{K}} \rightarrow B(M)$ naturally defined by $\widetilde{\kappa}\left(\mu+I_{\kappa}\right):=\kappa(\mu)$ is a continuous one to one homomorphism, where $I_{\mathcal{\kappa}}=\left\{\mu \in M_{0}(K): \kappa(\mu)=0\right\}$. ( $I_{\mathcal{\kappa}}$ clearly is a closed ideal of $M_{0}(K)$.)

Since $0 \rightarrow I_{\mathcal{K}} \rightarrow M_{0}(K) \rightarrow M_{0}(K) / I_{\mathcal{K}} \rightarrow 0$ is an exact sequence, by [18, page 324] we have

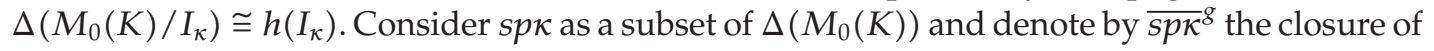


$s p \kappa$ in Gelfand topology. By regularity of $M_{0}(K)$, Gelfand and hull-kernel topologies coincide on $\Delta\left(M_{0}(K)\right)$. So $\overline{s p \kappa}^{g}$ is closed in hull-kernel topology. But the only hull-kernel-closed subsets of $\Delta\left(M_{0}(K)\right)$ are hull-sets. Then, there exists a subset $I \subseteq M_{0}(K)$ such that ${\overline{s p \kappa^{g}}}^{g}=h(I)$. Therefore,

$$
h k\left(\overline{s p \kappa}^{g}\right)=h k h(I)=h(I)={\overline{s p \kappa^{g}}}^{g} .
$$

On the other hand, for every $\mu \in M_{0}(K), \mu \in k\left(\overline{s p \kappa}^{g}\right)$ if and only if $T \mu=0$ on $\overline{s p \kappa}^{g}$. This is also equivalent to vanishing $\widehat{\mu}$ on spк. By Lemma 3.3, this holds if and only if $\kappa(\mu)=0$. Therefore, $h\left(I_{\mathcal{K}}\right)=\overline{s p \kappa}^{g}$.

By the regularity of $M_{0}(K)$, the Banach algebra $M_{0}(K) / I_{\mathcal{K}}$ is also regular. For any $\mu \in M_{0}(K), \mu+I_{\kappa} \in \operatorname{rad}\left(M_{0}(K) / I_{\kappa}\right)$ if and only if $\widehat{\mu+I_{\kappa}}=0$ on $\Delta\left(M_{0}(K) / I_{\kappa}\right)$. This is also equivalent to vanishing $T \mu$ on $h\left(I_{\kappa}\right)$. By the previous paragraph, this holds if and only if $\kappa(\mu)=0$, that is, $\mu \in I_{\kappa}$. Therefore, $M_{0}(K) / I_{\kappa}$ is a semisimple algebra.

In the sequel, we consider two cases.

Case 1. Let $\overline{s p \kappa}^{g}$ be compact. There exists $v \in M_{0}(K)$ such that $T v=1$ on a neighborhood $\overline{s p \kappa}^{g}$. Then, for any $\mu \in M_{0}(K)$ we have

$$
T(\mu * v-\mu)=T \mu T v-T \mu=0 \quad \text { on } \overline{s p \kappa}^{g} .
$$

So $\widehat{\mu * v-\mu}=0$ on $s p \kappa$ and by Lemma $3.3 \mu * v-\mu \in I_{\mathcal{K}}(\star)$. Then, for any $\mu \in M_{0}(K),\left(\mu+I_{\kappa}\right)(v+$ $\left.I_{\kappa}\right)=\mu+I_{\kappa}$. By $(\star)$, for any $f \in L^{1}(K)$,

$$
0=\kappa(v * f-f)=\kappa(v) \kappa(f)-\kappa(f)=\left(\kappa(v)-I_{B(M)}\right) \cdot \kappa(f) .
$$

That is $\left(\kappa(v)-I_{B(M)}\right) \cdot \mathcal{\kappa}\left(L^{1}(K)\right)=0$. Now, since $\kappa\left(L^{1}(K)\right)$ is without order, $\kappa(v)=I_{B(M)}$. Therefore, $\widetilde{\kappa}\left(v+I_{\kappa}\right)=I_{B(M)}$. Now, we are in a position using Lemma 3.2(i) for $\tilde{\kappa}, M_{0}(K) / I_{\mathcal{\kappa}}$ and $B(M)$. Then, for every $\mu \in M_{0}(K)$,

$$
\sigma_{B(M)}\left(\tilde{\kappa}\left(\mu+I_{\kappa}\right)\right)=\widehat{\mu+I_{\kappa}}\left(\Delta\left(\frac{M_{0}(K)}{I_{\kappa}}\right)\right),
$$

and so

$$
\sigma_{B(M)}(\kappa(\mu))=T \mu\left({\overline{s p \kappa^{g}}}^{g} .\right.
$$

Now compactness of $\overline{s p \kappa}^{g}$ in $\Delta\left(M_{0}(K)\right)$ and continuity of $T \mu$ on $\Delta\left(M_{0}(K)\right)$ imply that $T \mu\left(\overline{s p \kappa}^{g}\right)$ is compact and so is closed. This fact by using relation $\widehat{\mu}(s p \kappa)=T \mu(s p \kappa) \subseteq T \mu\left(\overline{s p \kappa}^{g}\right)$ shows that

$$
\overline{\widehat{\mu}(s p \kappa)} \subseteq T \mu\left(\overline{s p \kappa}^{g}\right) .
$$

On the other hand, since $T \mu$ is continuous, we have

$$
T \mu\left({\overline{s p \kappa^{g}}}^{g} \subseteq \overline{T \mu(s p \kappa)}=\overline{\widehat{\mu}(s p \kappa)} .\right.
$$

Thus,

$$
\overline{\widehat{\mu}(s p \kappa)}=T \mu\left({\overline{s p \kappa^{g}}}^{g} .\right.
$$

Now by $(\star \star), \sigma_{B(M)}(\kappa(\mu))=\overline{\widehat{\mu}(s p \kappa)}$. 
Case 2. Let ${\overline{s p \mathcal{K}^{g}}}^{g}$ be noncompact. Then, by $\Delta\left(M_{0}(K) / I_{\mathcal{K}}\right) \cong{\overline{s p \mathcal{K}^{g}}}^{g}, M_{0}(K) / I_{\mathcal{K}}$ is not unitary. So by Lemma 3.2(ii), for any $\mu \in M_{0}(K)$,

$$
\sigma_{B(M)}\left(\tilde{\kappa}\left(\mu+I_{\kappa}\right)\right)=\overline{\widehat{\mu+I_{\kappa}}\left(\Delta\left(\frac{M_{0}(K)}{I_{\mathcal{\kappa}}}\right)\right)} .
$$

Now the inclusion $\widehat{\mu}(s p \kappa) \subseteq T \mu\left({\overline{s p \kappa^{g}}}^{g}\right)$ implies $\overline{\widehat{\mu}(s p \kappa)} \subseteq \overline{T \mu\left(\overline{s p \kappa}^{g}\right)}$. Also since $T \mu$ is continuous, we have $T \mu\left({\overline{s p \kappa^{g}}}^{g}\right) \subseteq \overline{\widehat{\mu}(s p \kappa)}$, and so that $\overline{T \mu\left(\overline{s p \kappa}^{g}\right)} \subseteq \overline{\widehat{\mu}(s p \kappa)}$. Therefore,

$$
\sigma_{B(M)}(\kappa(\mu))=\overline{T \mu\left(\overline{s p \kappa}^{g}\right)}=\overline{\widehat{\mu}(s p \kappa)} .
$$

\section{Examples}

In this section, we give some examples of hypergroups $K$ that are commutative strong hypergroups and $\widehat{K}=X_{b}(K)$ (we refer to these conditions by notation $(\wp)$ ). Observe that any locally compact Abelian group has these properties. Also, if $G$ is a locally compact Abelian group and $H$ is a compact subgroup of $\operatorname{Aut}(G)$, then the space $G_{H}$ containing all $H$ orbits is a commutative hypergroup satisfying in $(\wp)$. In fact, $\widehat{G_{H}} \cong(\widehat{G})_{H}$. We refer to [19] for more details. As another example, let $G$ be a group such that $G / Z$ is compact, where $Z=\{x \in G$ : for any $y \in G, x y=y x\}$. If $K$ is the hypergroup containing all conjugacy classes of $G$, then $K$ and its dual $\widehat{K}$ satisfy in (६) [6]. On the other hand, an interesting example of Naimark given in [1, Section 9.5] does not satisfy in conditions $(\wp)$.

\section{References}

[1] R. I. Jewett, "Spaces with an abstract convolution of measures," Advances in Mathematics, vol. 18, no. 1, pp. 1-101, 1975.

[2] C. F. Dunkl, "The measure algebra of a locally compact hypergroup," Transactions of the American Mathematical Society, vol. 179, pp. 331-348, 1973.

[3] R. Spector, "Aperçu de la théorie des hypergroupes," in Analyse Harmonique sur les Groupes de Lie (Sém. Nancy-Strasbourg, 1973-75), vol. 497 of Lecture Notes in Mathematics, pp. 643-673, Springer, Berlin, Germany, 1975.

[4] B. R. Li, Introduction to Operator Algebras, World Scientific, River Edge, NJ, USA, 1995.

[5] J. B. Conway, A Course in Functional Analysis, vol. 96 of Graduate Texts in Mathematics, Springer, New York, NY, USA, 1985.

[6] W. R. Bloom and H. Heyer, Harmonic Analysis of Probability Measures on Hypergroups, vol. 20 of De Gruyter Studies in Mathematics, Walter de Gruyter, Berlin, Germany, 1995.

[7] R. Spector, "Mesures invariantes sur les hypergroupes," Transactions of the American Mathematical Society, vol. 239, pp. 147-165, 1978.

[8] A. R. Medghalchi and S. M. Tabatabaie, "Spectral subspaces on hypergroup algebras," submitted.

[9] A. Connes, "Une classification des facteurs de type III," Annales Scientifiques de l'École Normale Supérieure, vol. 6, pp. 133-252, 1973.

[10] C. D'Antoni, R. Longo, and L. Zsido, "A spectral mapping theorem for locally compact groups of operators," Pacific Journal of Mathematics, vol. 103, no. 1, pp. 17-24, 1982.

[11] J. Eschmeier, "Operator decomposability and weakly continuous representations of locally compact abelian groups," Journal of Operator Theory, vol. 7, no. 2, pp. 201-208, 1982.

[12] S.-E. Takahasi and J. Inoue, "A spectral mapping theorem for some representations of compact abelian groups," Proceedings of the Edinburgh Mathematical Society, vol. 35, no. 1, pp. 47-52, 1992. 
[13] H. Seferoğlu, "Spectral mapping theorem for representations of measure algebras," Proceedings of the Edinburgh Mathematical Society, vol. 40, no. 2, pp. 261-266, 1997.

[14] R. Larsen, An Introduction to the Theory of Multipliers, vol. 175 of Die Grundlehren der Mathematischen Wissenschaften, Springer, New York, NY, USA, 1971.

[15] A. K. Chilana and K. A. Ross, "Spectral synthesis in hypergroups," Pacific Journal of Mathematics, vol. 76, no. 2, pp. 313-328, 1978.

[16] R. Larsen, Banach Algebras: An Introduction, Pure and Applied Mathematics, no. 24, Marcel Dekker, New York, NY, USA, 1973.

[17] Y. Katznelson, An Introduction to Harmonic Analysis, Dover, New York, NY, USA, 1976.

[18] T. W. Palmer, Banach Algebras and the General Theory of *-Algebras. Vol. II, *-Algebras, vol. 79 of Encyclopedia of Mathematics and Its Applications, Cambridge University Press, Cambridge, UK, 2001.

[19] K. A. Ross, "Centers of hypergroups," Transactions of the American Mathematical Society, vol. 243, pp. 251-269, 1978. 


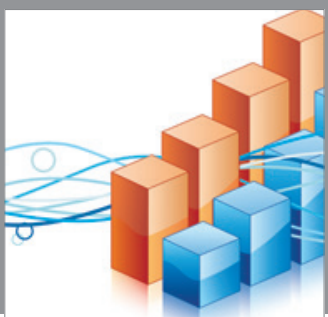

Advances in

Operations Research

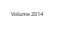

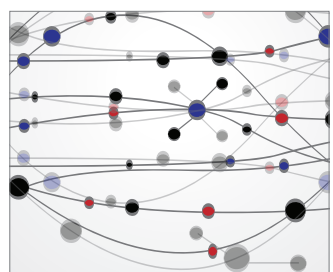

\section{The Scientific} World Journal
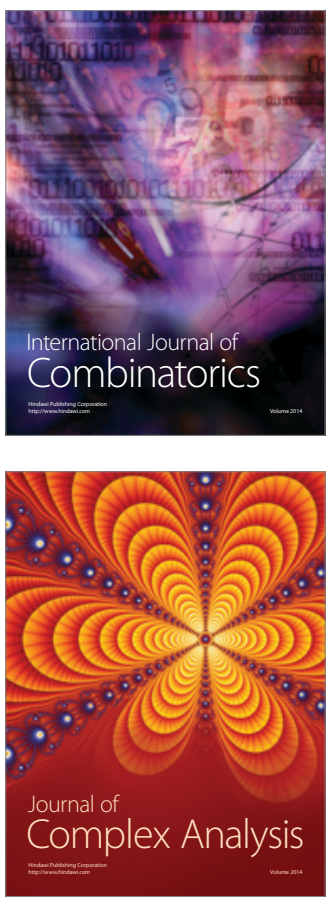

International Journal of

Mathematics and

Mathematical

Sciences
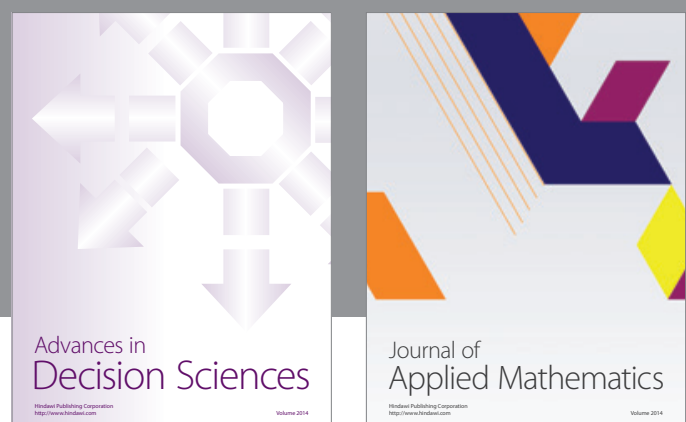

Journal of

Applied Mathematics
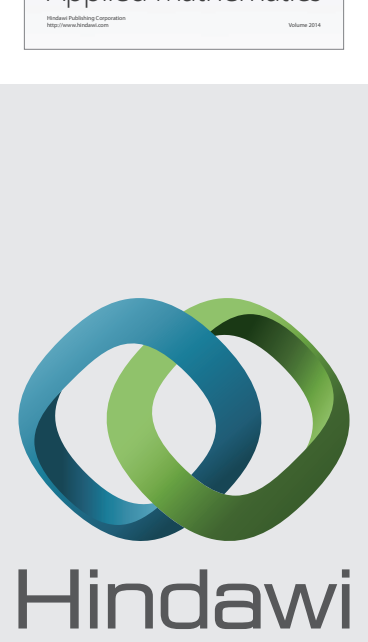

Submit your manuscripts at http://www.hindawi.com
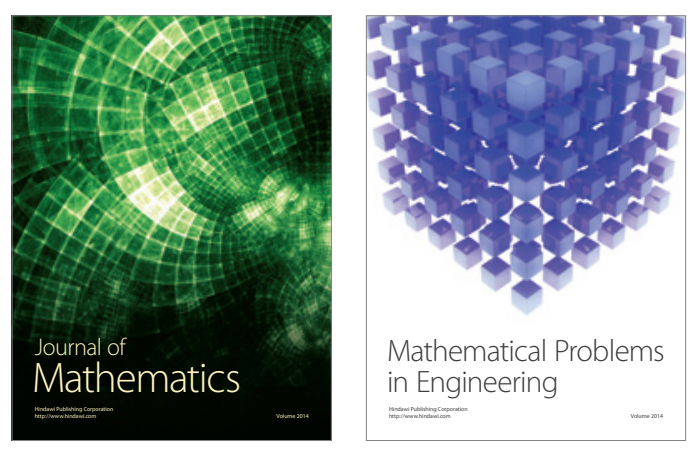

Mathematical Problems in Engineering
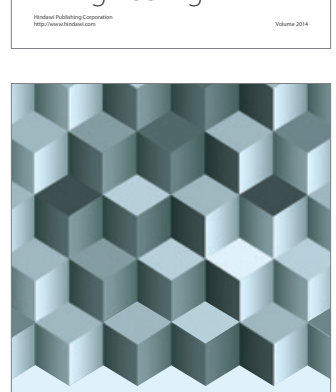

Journal of

Function Spaces
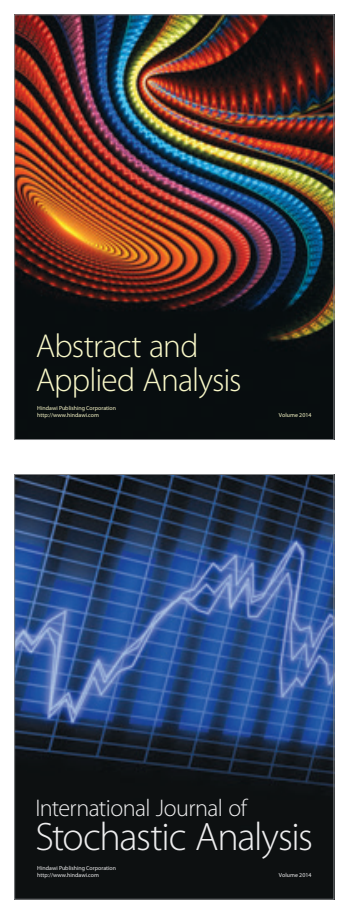

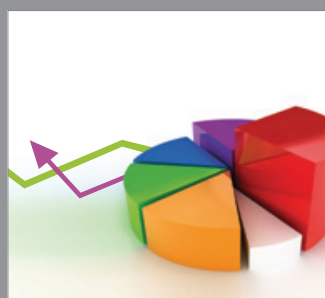

ournal of

Probability and Statistics

Promensencen
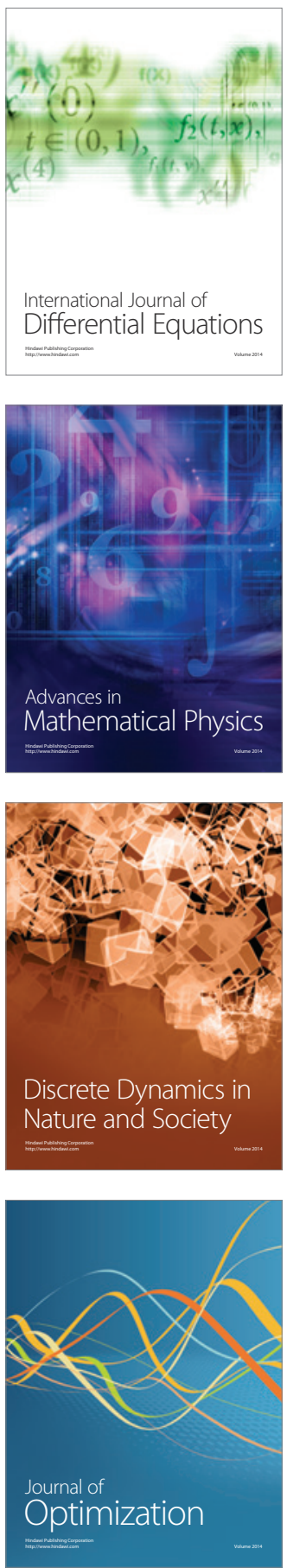